\title{
SOME CONSEQUENCES OF HARISH-CHANDRA'S SUBMERSION PRINCIPLE
}

\author{
CARY RADER AND ALLAN SILBERGER \\ (Communicated by Ronald M. Solomon)
}

\begin{abstract}
Let $G$ be a reductive p-adic group, $K$ a good maximal compact subgroup, $K_{1} \subset K$ any open subgroup, and $\pi$ an admissible representation of $G$ of finite type. In $A$ submersion principle and its applications, HarishChandra proves the theorem that $\int_{K} \pi\left(k g k^{-1}\right) d k$ is a finite-rank operator for $g$ in the regular set $G^{\prime}$ in order to show that the character $\Theta_{\pi}(g)$ is a locally constant class function on $G^{\prime}$. From this, the authors derive the formula $\theta(1) \boldsymbol{\Theta}_{\pi}(g)=d(\pi) \int_{G / Z} \int_{K_{1}} \theta\left(x k g k^{-1} x^{-1}\right) d k d \dot{x} \quad\left(g \in G^{\prime}\right)$ for any $K$-finite matrix coefficient $\theta$ of a discrete series representation $\pi$ with formal degree $d(\pi)$. They use another technical result of the paper to prove that invariant integrals of Schwartz space functions converge absolutely. None of these results depends upon a characteristic zero assumption.
\end{abstract}

\section{INTRODUCTION}

Let $\mathbb{F}$ be a commutative $p$-field, $G$ the group of $\mathbb{F}$-points of a connected reductive $\mathbb{F}$-group $\mathbf{G}$, and $G^{\prime}$ the set of regular (semisimple) points of $G$.

There is a well-known integral formula, proved originally by Harish-Chandra [4, pp. 60, 94] and rederived and used by Kutzko [6] (cf. also [7, Theorems 1.7 and 1.9]), which allows one in principle to compute the values of the character of a supercuspidal representation of $G$ on $G^{\prime}$, either by integrating a matrix coefficient of the representation or the character of a $K$-type which induces the representation. One purpose of this paper is to point out that this integral formula is actually valid for any discrete series representation of $G$ and, in this context, to give a new and simple proof of the integral formula based on Harish-Chandra's elegant paper [3].

In order not to obtain an unnecessarily restrictive special case of the integral formula for discrete series, the authors found it necessary to sharpen the statement of [3, Theorem 2]. The restatement appears here in $\S 2$ as Theorem 1; the modification to Harish-Chandra's argument is given in $\S 4$. Theorem 2 of $\S 2$ presents the integral formula for the character of a discrete series representation; the derivation of this formula from Theorem 1 is also given in $\S 2$.

Received by the editors August 26, 1991.

1991 Mathematics Subject Classification. Primary 22E50.

Key words and phrases. Character, discrete series, reductive $\mathfrak{p}$-adic groups, Schwartz space, invariant integral. 
Although he does not present the details, Harish-Chandra mentions that [ 3 , Theorem 3] can be used to give a characteristic-free proof of the absolute convergence of invariant integrals for Schwartz space functions. In $\S 3$, Theorem 4, we use [3, Theorem 4] to prove this absolute convergence. Our proof, while reminiscent of the argument presented in [9, p. 244] for real reductive Lie groups, simplifies Wallach's approach through the use of the "numerical function" of Geometric Invariant Theory from Kempf [5] and Mumford. In Corollary 5 we use Theorem 4 to express the discrete series characters on elliptic Cartan subgroups as invariant integrals of their matrix coefficients. It is interesting that the integral formula from Theorem 2 has support on all Cartan subgroups, whereas the invariant integrals vanish on the Cartan subgroups which are not elliptic. Related work also appears in Clozel [1].

\section{THE INTEgRAL FORMULA FOR THE CHARACTER OF A DISCRETE SERIES REPRESENTATION}

We use the notational conventions of $[2,3,8]$. In particular, for $\mathbf{X}$ an $\mathbb{F}$ group, we write $X$ to denote its corresponding group of $\mathbb{F}$-points.

Let $Z$ denote the split component of $G$. Let $(P, A)(P=M N)$ denote a minimal $p$-pair of $G$ and $K$ an $A$-special maximal compact subgroup of $G$. In the following $K_{1}$ denotes an arbitrary open subgroup of $K$. Fix Haar measures $d g$ on $G$ and $d \dot{g}$ on $G / Z$ such that $\int_{K_{1}} d g=\int_{K_{1} / Z \cap K_{1}} d \dot{g}=1$.

Let $\pi$ be an admissible representation of $G$ acting in a complex vector space $V$. Let $C_{c}^{\infty}(G)$ denote the convolution algebra of compactly supported locally constant functions on $G$. For any $f \in C_{c}^{\infty}(G)$

$$
\pi(f)=\int_{G} f(g) \pi(g) d g
$$

is an operator of finite rank acting in $V$. The mapping

$$
f \mapsto \Theta_{\pi}(f)=\operatorname{tr}(\pi(f)) \quad\left(f \in C_{c}^{\infty}(G)\right)
$$

is the (distributional) character of $\pi$. Harish-Chandra has proved in [3] that if $V$ is a module of finite type under $C_{c}^{\infty}(G)$, then there is a locally constant function $\Theta_{\pi}(g)$ defined for all $g \in G^{\prime}$ such that

$$
\Theta_{\pi}(f)=\int_{G^{\prime}} f(g) \Theta_{\pi}(g) d g
$$

for all $f \in C_{c}^{\infty}(G)$ with support in $G^{\prime}$. His proof depends upon the following assertion, proved in [3] only for the case $K_{1}=K$ :

Theorem 1 (Harish-Chandra). Assume that $\pi$ is an admissible representation of $G$ in a complex vector space $V$ and that $V$ is a left $C_{c}^{\infty}(G)$-module of finite type under $\pi$. Then

$$
g \mapsto \int_{K_{1}} \pi\left(k g k^{-1}\right) d k \quad\left(g \in G^{\prime}\right)
$$

is a locally constant function with range in the space of finite rank operators on $V$.

As we shall need Theorem 1 for arbitrary $K_{1}$, we shall indicate the modification in Harish-Chandra's argument needed to prove the more general version in $\S 4$. 
For the remainder of $\S 2$ assume Theorem 1 , as stated. Let $\pi$ now be an irreducible, admissible discrete series representation which is unitary on the pre-Hilbert space $V$. Write $\langle u, v\rangle$ for the inner product of $u, v \in V$. Let $\mathscr{A}(\pi)$ denote the vector space spanned by functions of the form

$$
x \mapsto\langle\pi(x) u, v\rangle \quad(x \in G ; u, v \in V) .
$$

With respect to the fixed Haar measure $d \dot{x}$ on $G / Z$ the formal degree $d(\pi)$ of $\pi$ is defined such that

$$
d(\pi)^{-1}\left\langle u_{1}, u_{2}\right\rangle \operatorname{conj}\left\langle v_{1}, v_{2}\right\rangle=\int_{G / Z}\left\langle\pi(x) u_{1}, v_{1}\right\rangle \operatorname{conj}\left\langle\pi(x) u_{2}, v_{2}\right\rangle d \dot{x} .
$$

Theorem 2. Let $\theta(x) \in \mathscr{A}(\pi)$ and let $g \in G^{\prime}$. Then

$$
\theta(1) \Theta_{\pi}(g)=d(\pi) \int_{G / Z} \int_{K_{1}} \theta\left(x k g k^{-1} x^{-1}\right) d k d \dot{x} .
$$

Remark. Since $\int_{G / Z \times K_{1}}\left|\theta\left(x k g k^{-1} x^{-1}\right)\right| d \dot{x} \times d k$ does not exist, in general, it is not possible to use the right invariance of the Haar measure $d \dot{x}$ to absorb the integration over $K_{1}$ into the integration over $G / Z$. Indeed, without the integration over $K_{1}$, the integrand would be constant on cosets of the centralizer of $g$; the centralizer of $g$ not being compact (when $g$ is not an elliptic element), the integral would diverge trivially.

Proof. Since the operator

$$
T_{g}=\int_{K_{1}} \pi\left(k g k^{-1}\right) d k
$$

has finite rank, there exists an open normal subgroup $\widetilde{K} \subset K_{1}$ such that

$$
\pi\left(k_{1}\right) T_{g} \pi\left(k_{2}\right)=T_{g}
$$

for all $k_{1}, k_{2} \in \tilde{K}$. Let $V_{2}$ denote the subspace consisting of all $\tilde{K}$-fixed vectors in $V$. By the choice of $\widetilde{K}$ we may assume $\operatorname{dim}\left(V_{2}\right)>0$. Choose an orthonormal basis $\left\{e_{i}\right\}$ for $V$ such that $e_{1}, \ldots, e_{N}$ is an orthonormal basis for $V_{2}$. Without loss of generality we assume that $\theta(g)=\langle\pi(g) u, v\rangle$ for some $u, v \in V$. Then

$$
\begin{aligned}
\int_{K_{1}} \theta\left(x k g k^{-1} x^{-1}\right) d k & =\int_{K_{1}}\left\langle\pi\left(k g k^{-1}\right) \pi\left(x^{-1}\right) u, \pi\left(x^{-1}\right) v\right\rangle d k \\
& =\sum_{i, j=1}^{N}\left\langle T_{g} e_{i}, e_{j}\right\rangle\left\langle\pi\left(x^{-1}\right) u, e_{i}\right\rangle \operatorname{conj}\left\langle\pi\left(x^{-1}\right) v, e_{j}\right\rangle \\
& =\sum_{i, j=1}^{N}\left\langle T_{g} e_{i}, e_{j}\right\rangle\left\langle\pi(x) e_{j}, v\right\rangle \operatorname{conj}\left\langle\pi(x) e_{i}, u\right\rangle .
\end{aligned}
$$

Since, for the discrete series representation $\pi$,

$$
\int_{G / Z}\left\langle\pi(x) e_{j}, v\right\rangle \operatorname{conj}\left\langle\pi(x) e_{i}, u\right\rangle d \dot{x}=d(\pi)^{-1}\left\langle e_{j}, e_{i}\right\rangle\langle u, v\rangle=d(\pi)^{-1} \delta_{i j} \theta(1)
$$


and since we can interchange $\int_{G / Z}$ and the finite summation $\sum_{i, j=1}^{N}$, we obtain

$$
\begin{aligned}
d(\pi) \int_{G / Z} \int_{K_{1}} \theta\left(x k g k^{-1} x^{-1}\right) d k d \dot{x} & =d(\pi) \sum_{i, j=1}^{N}\left\langle T_{g} e_{i}, e_{j}\right\rangle d(\pi)^{-1} \delta_{i j} \theta(1) \\
& =\operatorname{tr}\left(T_{g}\right) \theta(1) .
\end{aligned}
$$

Finally, if $f \in C_{c}^{\infty}\left(G^{\prime}\right)$, then

$$
\begin{aligned}
\Theta_{\pi}(f) & =\operatorname{tr}\left(\int_{G^{\prime}} f(g) \pi(g) d g\right)=\operatorname{tr}\left(\int_{G^{\prime}} \int_{K_{1}} f\left(k^{-1} g k\right) d k \pi(g) d g\right) \\
& =\operatorname{tr}\left(\int_{G^{\prime}} f(g) \int_{K_{1}} \pi\left(k g k^{-1}\right) d k d g\right)=\int_{G^{\prime}} f(g) \operatorname{tr}\left(T_{g}\right) d g .
\end{aligned}
$$

This concludes the proof of Theorem 2 .

\section{ON THE INVARIANT INTEgRALS OF SCHWARTZ FUNCTIONS}

In this section we use [3] to construct a proof of the convergence of invariant integrals for Schwartz functions. Let

$$
\varphi: G \rightarrow \mathrm{GL}_{n}(\mathbb{F})
$$

be an irreducible faithful rational representation of $G$ on $V=\mathbb{F}^{n}$, defined over $\mathbb{F}$. For $T \in \mathscr{M}_{n}(\mathbb{F})$ (the space of $n$ by $n$ matrices over $\mathbb{F}$ ) define

$$
\|T\|=\max _{i, j}\left|T_{i j}\right|
$$

and for $x \in G$ define

$$
\|x\|=\inf _{z \in Z} \max \left(\|\varphi(x z)\|,\left\|\varphi(x z)^{-1}\right\|\right) .
$$

Define the relations $\prec$ and $\asymp$ as in [8, p. 149]. In [3, p. 101] Harish-Chandra defines

$$
\beta(g)=\sup _{x}\left(f_{\alpha_{1}, g}(x)\right) \quad\left(g \in G^{\prime}, K_{1}=K\right) .
$$

Let $\Gamma$ be an elliptic Cartan subgroup of $G$, let $\Gamma^{\prime}=\Gamma \cap G^{\prime}$ denote the set of regular elements in $\Gamma$, and let $\Xi$ denote the spherical function used in Harish-Chandra's definition of the Schwartz space for $G([2, \S 14]$ or $[8$, $\S 4.2])$.

Lemma 3. Let $\omega$ be a compact subset of $\Gamma^{\prime}$. Then there are positive constants $c$ and $r$ such that, for any $g \in \omega$,

$$
\int_{K} \Xi\left(m^{-1} k^{-1} g k m\right) d k \leq c \beta(g) \Xi(m)^{2}
$$

and

$$
\left\|m^{-1} k^{-1} g k m\right\| \geq c^{-1}\|m\|^{r}
$$

for all $m \in M$.

Proof. A stronger version of (1) is proved in [3, p. 101] (where $\omega \subset \Gamma^{\prime}$ only has to be precompact in $\Gamma$ and $\Gamma$ need not be elliptic). (Harish-Chandra ostensibly proves the assertion of (1) only for $m \in M^{+}$, but one can use the $K$-invariance 
of $\Xi$ to obtain his assertion for any $m \in M$.) We prove only (2), using an idea from [5]. The assertion (2) factors through $G / Z$, so for the rest of the proof we assume that $Z=\{1\}$. Set

$$
\Omega=\left\{k^{-1} g k: k \in K \text { and } g \in \omega \cup \omega^{-1}\right\},
$$

a compact set of elliptic regular points of $G$. Let $\Lambda$ be the set of weights of $\varphi$ for $A$, the split component of $M$, and let $E_{\lambda}(\lambda \in \Lambda)$ be the corresponding projection onto the $\lambda$-eigenspace $V_{\lambda}$. Without loss of generality we assume that for $\gamma \in \Omega$ and $m \in M$,

$$
\begin{aligned}
\left\|\varphi\left(m^{-1} \gamma m\right)\right\| & =\max \left\{\left\|E_{\lambda} \varphi\left(m^{-1} \gamma m\right) E_{\nu}\right\|: \lambda, \nu \in \Lambda\right\} \\
& \asymp \max \left\{q^{\langle\nu-\lambda, H(m)\rangle}\left\|E_{\lambda} \varphi(\gamma) E_{\nu}\right\|: \lambda, \nu \in \Lambda\right\} .
\end{aligned}
$$

For $m \in M$ with $m \notin{ }^{0} M[8$, p. 8] we have the flag in $V$,

$$
F_{\lambda}=\bigoplus\left\{V_{\nu}:\langle\nu, H(m)\rangle \leq\langle\lambda, H(m)\rangle\right\} \quad(\nu, \lambda \in \Lambda) .
$$

Let $P_{m}=M_{m} N_{m}$ be the proper parabolic subgroup in $G$ which stabilizes this flag, so $\varphi\left(M_{m}\right)$ consists of block diagonal matrices and $\varphi\left(N_{m}\right)$ of block upper triangular matrices with respect to $F_{\lambda}$. Thus

$$
P_{m}=\left\{x \in G: E_{\lambda} \varphi(x) E_{\nu}=0 \text { if }\langle\nu, H(m)\rangle \geq\langle\lambda, H(m)\rangle\right\} .
$$

Define

$$
l(m, \gamma)=\max \left\{\langle\nu-\lambda, H(m)\rangle:\left\|E_{\lambda} \varphi(\gamma) E_{\nu}\right\| \neq 0\right\}
$$

for $m \in M$ with $m \notin{ }^{0} M$ and $\gamma \in \Omega$ (cf. [5, p. 306]). Clearly, if $l(m, \gamma) \leq 0$, then $\gamma \in P_{m}$ (cf. [5, p. 305]); conjugating by $N_{m}$, we may assume that $\gamma \in M_{m}$. It follows that

$$
\Gamma=\operatorname{cent}(\gamma)^{0} \supset A_{m}=\text { split center of } M_{m},
$$

which is impossible since $\Gamma$ is elliptic. Set

$$
L(m, \gamma)=\max \left\{\left\|E_{\lambda} \varphi(\gamma) E_{\nu}\right\|:\langle\nu-\lambda, H(m)\rangle=l(m, \gamma)\right\} .
$$

Then

$$
\left\|\varphi\left(m^{-1} \gamma m\right)\right\| \geq L(m, \gamma) q^{l(m, \gamma)} \quad(\gamma \in \Omega, m \in M) .
$$

Next note that $L$ and $l$ are strictly positive (for $m \notin{ }^{0} M$ ) locally constant functions. As $\gamma$ varies over the compact set $\Omega$, the set of values assumed by $L(m, \gamma)$ is a finite set of positive numbers; let $L$ be the smallest of these. Since the $\lambda-\nu$ are integral linear combinations of roots, it is clear that $l$ extends to the real Lie algebra,

$$
l: \mathfrak{a}_{\mathbb{R}} \times \Omega \rightarrow \mathbb{R}_{+}
$$

as a continuous function which is convex and positively homogeneous of degree one on $\mathfrak{a}_{\mathbb{R}}$ and locally constant on $\Omega$.

On the other hand, if $H(a)$ lies in a closed positive Weyl chamber $\mathfrak{a}^{+}$and $\lambda$ is the highest weight for $\varphi$ relative to $\mathfrak{a}^{+}$, then $\|a\|=q^{\langle\lambda, H(a)\rangle}$ relative to a basis of eigenvectors for $A$, since the other weights are of the form $\lambda-\sum m_{t r} \alpha$ with integers $m_{\alpha} \geq 0$ and $\alpha \geq 0$ on $A^{+}$. Thus

$$
a \mapsto \sigma(a)=\max \{\langle\lambda, H(a)\rangle: \lambda \text { is an extreme weight of } \varphi\}
$$


extends to a continuous, convex function on $\mathfrak{a}_{\mathbb{R}}$ which is positively homogeneous of degree one and strictly positive away from zero (strictly positive because $\varphi$ is faithful). Let $\mathscr{S}$ be the unit sphere in $\mathfrak{a}_{\mathbb{R}}$. Then

$$
r=\inf \{l(H, \gamma) / \sigma(H):(H, \gamma) \in \mathscr{S} \times \Omega\}
$$

is positive, and

$$
\left\|\varphi\left(m^{-1} \gamma m\right)\right\| \geq L q^{l(m, \gamma)} \geq L q^{r \sigma(H(m))} \succ L\|m\|^{r}
$$

for $\gamma \in \Omega$. But also $\gamma^{-1} \in \Omega$, so $\left\|m^{-1} \gamma m\right\| \succ\|m\|^{r}$. This completes the proof of the lemma.

Recall that the Schwartz space $\mathscr{C}(G)$ is the space of functions $f$ on $G$ such that $f \in C\left(G / / K_{0}\right)$, the space of $K_{0}$-bi-invariant functions, for some compact open subgroup $K_{0} \subset G$, and that

$$
|f|_{N}=\sup _{x \in G}|f(x)| \Xi(x)^{-1}(1+\log \|x\|)^{N}
$$

is finite for each $N \in \mathbb{N}$. If $\Gamma$ is a Cartan subgroup of $G, A_{\Gamma}$ is its split component, and $f \in \mathscr{C}(G)$, then

$$
F_{f}(g)=|D(g)|^{1 / 2} \int_{G / A_{\Gamma}} f\left(x g x^{-1}\right) d x^{*} \quad\left(g \in \Gamma^{\prime}\right)
$$

is called the invariant integral. Here $D(g)$ is the lowest coefficient in the characteristic polynomial of $\operatorname{Ad}(g)-1$ and $d x^{*}$ is the invariant measure on $G / A_{\Gamma}$.

Theorem 4. There exists an integer $N$ with the following property. For any compact set $\omega \subset \Gamma^{\prime}$ there is a constant $C>0$ such that for all $f \in \mathscr{C}(G)$

$$
\left|F_{f}(g)\right| \leq C|f|_{N}
$$

for all $g \in \omega$. Moreover, $F_{f}$ is locally constant on $\Gamma^{\prime}$ (for every $f \in \mathscr{C}(G)$ ), and for a fixed compact open subgroup $K_{0} \subset G$, the space of restrictions

$$
\left\{F_{f} \mid \omega: f \in \mathscr{C}\left(G / / K_{0}\right)\right\}
$$

is a finite-dimensional vector space.

Proof. If $\Gamma$ is not elliptic, then choose a parabolic subgroup $P=M N$ so that $A=A_{\Gamma}$ is the split component of $M$, and $\Gamma \subset M$ is elliptic. Then we have the continuous map

$$
f \mapsto f^{P}: \mathscr{C}(G) \rightarrow \mathscr{C}(M), \quad\left|f^{P}\right|_{M, n} \leq C_{n}|f|_{G, n+d_{A}}
$$

(for some integer $d_{A}$ and all integers $n[8$, p. 176]) which satisfies

$$
F_{f}^{G / \Gamma}(g)=F_{\bar{f}^{p}}^{M / \Gamma}(g) \quad\left(g \in \Gamma^{\prime}\right)
$$

(where $\bar{f}(x)=\int_{K} f\left(k x k^{-1}\right) d k$ ) [4, p. 58].

This reduces the proof to the case of an elliptic Cartan subgroup $\Gamma$. We use the Cartan integration formula [8, p. 149]. For $g \in \omega$ (and letting $\mu$ denote 
our normalized Haar measure)

$$
\begin{aligned}
|D(g)|^{-1 / 2}\left|F_{f}^{\Gamma}(g)\right| & f\left(l^{-1} m^{-1} k^{-1} g k m l\right) \mu(K m K) d k d m d l \mid \\
& =\mid \int_{K \times M^{+} \times K} \Xi\left(m^{-1} k^{-1} g k m\right)\left(1+\log \left\|m^{-1} k^{-1} g k m\right\|\right)^{-n} \mu(K m K) d k d m \\
& \prec \int_{M^{+} \times K}(1+r \log \|m\|)^{-n} \delta_{P_{0}}(m) \int_{K} \Xi\left(m^{-1} k^{-1} g k m\right) d k d m \\
& \prec \int_{M^{+}}(\text {Lemma } 3 \text { and [8, Lemma 4.1.1]) } \\
& \prec \beta(g) \int_{M^{+}}(1+r \log \|m\|)^{-n} \delta_{P_{0}}(m) \Xi(m)^{2} d m \quad \text { (Lemma 3) } \\
& \prec \beta(g) \sum_{M^{+} /{ }^{0} M}(1+r \log \|m\|)^{-n+2 r_{0}} \quad[8, \mathrm{p} .154] \\
& \prec \beta(g) \quad[8, \mathrm{p} .150] .
\end{aligned}
$$

This implies the first sentence of the theorem. The rest comes directly from [3, Theorem 3] and the fact that $C_{c}\left(G / / K_{0}\right)$ is dense in $\mathscr{C}\left(G / / K_{0}\right)$.

As a corollary to the last result, note that if $\Gamma$ is elliptic (so $A_{\Gamma}=Z$ ), the integral in Theorem 2 is absolutely convergent, since $\theta$ is a matrix entry of a discrete series representation, so lies in $\mathscr{C}(G)$. Thus we can reverse the order of integration and absorb the integration over $K$ into the Haar invariance of $d \dot{x}$. Moreover, since $\theta$ is a cusp form, we obtain

Corollary 5. Under the assumptions of Theorem 2, if $g \in G^{\prime}$ is regular elliptic, then

$$
d(\pi) \int_{G / Z} \theta\left(x g x^{-1}\right) d \dot{x}=\theta(1) \Theta_{\pi}(g),
$$

and if $g$ is regular but not elliptic and $\Gamma$ is the centralizer of $g$, then

$$
\int_{G / A_{\Gamma}} \theta\left(x g x^{-1}\right) d \dot{x}=0
$$

(cf. $[1, p .9])$.

\section{THE PROOF OF THEOREM 1}

We use notation like [3, p. 98], and where notation or terminology is not explained we have used Harish-Chandra's. (To read this proof it will be necessary to have Harish-Chandra's article close at hand.)

For simplicity assume that $K_{1}$ is a normal subgroup of $K$. There is no loss of generality in this assumption inasmuch as every open subgroup of $G$ contains an open normal subgroup of $K$. Moreover, if Theorem 1 is true for an open subgroup of $K_{1}$, then it is obviously true for $K_{1}$, too. Let $K_{1}, \ldots, K_{n}$ be the cosets of $K_{1}$ in $K$, and for $1 \leq i \leq n$ define

$$
T_{g, i}=\int_{K_{i}} \pi\left(k g k^{-1}\right) d k, \quad T_{g}=T_{g, 1} \quad\left(g \in G^{\prime}\right) .
$$


For every $k \in K$ there exists $i=i(k)$ such that

$$
T_{g} \pi(k)=\pi(k) T_{g, i} \quad\left(g \in G^{\prime}, k \in K\right) .
$$

Let $P_{0}$ be an open compact subgroup of $P$ chosen as at the top of [3, p. 99]. Let $\alpha_{i} \in C_{c}^{\infty}(G \times P)$ be the characteristic function of $K_{i} \times P_{0}$ for each $i$. HarishChandra's "submersion principle" applied to the submersion of $G \times P \rightarrow G$ defined by

$$
(x, p) \mapsto x g x^{-1} p \quad\left(\text { fixed } g \in G^{\prime}\right)
$$

implies the existence of functions $f_{\alpha_{i}, g} \in C_{c}^{\infty}(G)$ such that

$$
\begin{aligned}
\int_{K_{i} \times P_{0}} F\left(k g k^{-1} p\right) d k d_{l} p & =\int_{G \times P} \alpha_{i}(x, p) F\left(x g x^{-1} p\right) d x d_{l} p \\
& =\int_{G} f_{\alpha_{i}, g}(y) F(y) d y
\end{aligned}
$$

for any locally integrable function $F$ on $G\left(d_{l} p\right.$ denotes a left Haar measure on $P$ ). Let $V_{0}$ denote a finite-dimensional subspace of $V$ such that $\pi\left(C_{c}^{\infty}(G)\right) V_{0}=V$ and such that $V_{0}$ is the space of all $K_{0}$-fixed vectors for some open normal subgroup $K_{0}$ of $K$. Then for $F=\pi$ and on the vector space spanning set for $V$

$$
\left\{\pi(k m) v \mid k \in K, m \in M^{+}, v \in V_{0}\right\}
$$

we obtain the relation

$$
T_{g} \pi(k m) v=\pi(k) T_{g, i} \int_{P_{0}} \pi(p) d_{l} p \pi(m) v=\pi(k) \pi\left(f_{\alpha_{i}, g}\right) \pi(m) v,
$$

which implies that $T_{g}$ is an operator of finite rank. In fact, choosing an open normal subgroup $\widetilde{K} \subset K$ such that $f_{\alpha_{i}, g} \in C_{c}^{\infty}(G / / \widetilde{K})$ for each $i=1, \ldots, n$ we have

$$
\pi\left(E_{\widetilde{K}}\right) T_{g} \pi(k m) v=\pi(k) \pi\left(E_{\widetilde{K}} f_{\alpha_{i}, g}\right) \pi(m) v=T_{g} \pi(k m) v,
$$

where $E_{\widetilde{K}}$ is the identity element in the convolution algebra $C_{c}^{\infty}(G / / \widetilde{K})$. Assuming that $\widetilde{K} \subset K_{1}$ and using the fact that $T_{g}$ commutes with $\pi\left(K_{1}\right)$, we conclude that, since

$$
\pi\left(E_{\widetilde{K}}\right) T_{g}=T_{g} \pi\left(E_{\widetilde{K}}\right)=T_{g},
$$

$T_{g}$ is of finite rank. (Harish-Chandra also shows that $(g, x) \mapsto f_{\alpha_{i}, g}(x)$ lies in $C^{\infty}\left(G^{\prime} \times G\right)$ and is compactly supported in $x$. This implies that $T_{g, i}$ is locally constant in $g$.)

\section{REFERENCES}

1. Laurent Clozel, Invariant harmonic analysis on the Schwartz space of a reductive $\mathfrak{p}$-adic group, Harmonic Analysis on Reductive Groups (Bowdoin Conference), Birkhauser, Boston, MA, 1991, pp. 101-121.

2. Harish-Chandra, Harmonic analysis on reductive p-adic groups, Proc. Sympos. Pure Math., vol. 26, Amer. Math. Soc., Providence, RI, 1974, pp. 167-192.

3. K. Patodi, Indian Academy of Sciences, Bangalore, and the Tata Institute of Fundamental Research, Bombay, 1980, pp. 95-102. 
4. __ Harmonic analysis on reductive $\mathfrak{p}$-adic groups, Notes by G. van Dijk, Lecture Notes in Math., vol. 162, Springer-Verlag, Berlin, Heidelberg, and New York, 1970.

5. George Kempf, Instability in invariant theory, Ann. of Math. (2) 108 (1978), 299-316.

6. Philip Kutzko, Character formulas for supercuspidal representations of $G L_{l}, l$ a prime, Amer. J. Math. 109 (1987), 201-222.

7. Paul J. Sally, Jr., Some remarks on discrete series characters for reductive $\mathfrak{p}$-adic groups, Representations of Lie Groups, Kyoto, Hiroshima, 1986, Adv. Stud. Pure Math., vol. 14, North-Holland, Amsterdam and New York, 1988, pp. 337-348.

8. Allan J. Silberger, Introduction to harmonic analysis on reductive $\mathfrak{p}$-adic groups, Math. Notes, vol. 23, Princeton Univ. Press, Princeton, NJ, 1979.

9. Nolan Wallach, Real reductive groups. I, Academic Press, Boston, MA, 1988.

Department of Mathematics, Ohio State University, Newark, Ohio 43055

E-mail address: cbrader@mps.ohio-state.edu

Department of Mathematics, Cleveland State University, Cleveland, Ohio 44115

E-mail address: R0730@vmcms.csuohio.edu 